\title{
REVIEW
}

\section{High-Density Lipoprotein in Lupus: Disease Biomarkers and Potential Therapeutic Strategy}

\author{
Sang Yeop Kim, ${ }^{1}$ (D) Minzhi Yu, ${ }^{1}$ (D) Emily E. Morin, ${ }^{1}$ Jukyung Kang, ${ }^{1}$ Mariana J. Kaplan, ${ }^{2}$ and \\ Anna Schwendeman' ${ }^{1}$ D
}

Systemic lupus erythematosus (SLE) patients exhibit accelerated development of atherosclerosis and increased incidents of cardiovascular disease (CVD) that cannot be explained by traditional risk factors alone. Accumulating evidence suggests that reduced levels of high-density lipoproteins (HDLs), along with altered HDL composition and function, may contribute to the accelerated atherosclerosis in SLE patients. Normally, HDLs play various atheroprotective roles through facilitating cholesterol efflux, inhibiting vascular inflammation, and scavenging oxidative species. However, systemic inflammation, oxidative stress, and autoimmunity in SLE patients induce changes in HDL size distribution and proteomic and lipidomic signatures. These compositional changes in HDLs result in the formation of proinflammatory, dysfunctional HDL. These lupus-altered HDLs have impaired antiatherogenic function with reduced cholesterol efflux capacities, impaired antioxidation abilities, and diminished antiinflammatory properties. In fact, dysfunctional HDL may promote atherogenesis by inducing inflammation. Thus, dysfunctional HDLs could be an important biomarker of accelerated atherosclerosis in lupus. Additionally, HDL-targeted therapies, especially infusion of reconstituted HDLs, may serve as a potential therapeutic intervention for SLE patients with CVD.

\section{Introduction}

Systemic lupus erythematosus (SLE) is an autoimmune syndrome with pleiotropic clinical manifestations, characterized by the synthesis of autoantibodies, the development of significant immune dysregulation, and organ damage. While life expectancy in SLE has improved due to the advancement of immunosuppressive therapies and improved treatments for infections and renal disease, mortality rates remain $\sim 3$-fold higher than in the general population (1). Of the many causes of mortality in SLE, accelerated atherosclerosis and cardiovascular disease (CVD) is recognized as one of the most prevalent (2). SLE patients showed a higher prevalence of coronary artery disease and atherosclerosis compared to controls, which could not be predicted by traditional risk factors alone (3). Since CVD-inflicted mortality accounts for

Supported in part by the American Heart Association (grant 13SDG17230049), the University of Michigan (College of Pharmacy Upjohn Award and Mcubed), the NIH (grants R01-GM-113832 and R01-HL-134569), the Intramural Research Program of the National Institute of Arthritis and Musculoskeletal and Skin Diseases, NIH (grant ZIA-AR-041199), and the Lupus Research Institute. Ms Yu's work was supported by the American Heart Association (grant AHA 19PRE34400017). Dr. Morin's work was supported by the Cellular Biotechnology Training Program (T32-GM008353) and the Translational Cardiovascular Research and Entrepreneurship Training Program (grant T32-HL125242). more than one-third of all deaths in SLE patients (4), it is clear that CVD continues to be a significant threat to SLE patients.

High-density lipoprotein (HDL) is the smallest lipoprotein and is well-known to exhibit various atheroprotective effects independent of cholesterol mobilization, including its antioxidative, antiinflammatory, antithrombotic, and antiapoptotic abilities (5). Thus, low levels of HDL have been associated with an increased risk of CVD. SLE patients with accelerated atherosclerosis exhibit decreased levels of HDL and the development of dysfunctional HDL (6-8). Such evidence suggests that HDL is likely a novel target for minimizing the risk of CVD in SLE patients, and several studies have recently proposed and investigated HDLtargeted therapies as a potential therapeutic intervention in SLE patients with CVD. In this review, we will discuss the quantitative and qualitative roles of HDL under both normal conditions and

${ }^{1}$ Sang Yeop Kim, PhD, Minzhi Yu, MS, Emily E. Morin, PhD, Jukyung Kang, PhD, Anna Schwendeman, PhD: University of Michigan, Ann Arbor; ${ }^{2}$ Mariana J. Kaplan, MD: National Institute of Arthritis and Musculoskeletal and Skin Diseases, NIH, Bethesda, Maryland.

Dr. Kim and Ms Yu contributed equally to this work.

No potential conflicts of interest relevant to this article were reported.

Address correspondence to Anna Schwendeman, PhD, University of Michigan, North Campus Research Center, 2800 Plymouth Road, Ann Arbor, MI 48109. E-mail: annaschw@med.umich.edu.

Submitted for publication May 14, 2018; accepted in revised form July 23, 2019. 


\section{Highlights}

- There are quantitative and qualitative abnormalities in HDL of SLE patients including low HDL levels, altered HDL subclass distributions, proteomic and lipidomic changes, and presence of oxidized HDL particles.

- The cholesterol efflux, anti-inflammatory and anti-oxidative functions of HDL are impaired in SLE patients, possibly contributing to the accelerated atherosclerosis development.

- HDL targeted therapy which aims to restore levels and quality of HDL holds a potential in halting development of CVD in SLE patients.

Figure 1. A brief summary of the role of high-density lipoprotein (HDL) in systemic lupus erythematosus (SLE). CVD = cardiovascular disease.

in patients with SLE, as well as the potential of HDL-targeted therapeutic interventions (Figure 1).

\section{Structure and composition of HDLs}

HDL is constantly remodeled in the bloodstream through interactions with other lipoproteins, enzymes, and contact with target cells, resulting in significant particle heterogeneity. HDL consists of a core of hydrophobic lipids, including cholesteryl esters and triglycerides, and a surface monolayer containing phospholipids, free cholesterol, and apolipoproteins. Apolipoprotein A-I (Apo A-I) is the most abundant protein associated with HDL, comprising $70 \%$ of the total HDL protein content (9). Apo A-I is a 28.1-kd, highly a-helical and amphipathic scaffold protein consisting of 243 amino acids that interact with lipids to ultimately define the size and shape of HDL species (9).

In addition to proteins, lipid species are another key component of the overall structure of HDL. A recent lipidome analysis by Kontush et al revealed that more than half of the total HDL mass is accounted for by lipid components, the majority being phospholipids - accounting for $40-60 \%$ of the total lipid mass (10). Of these phospholipids, phosphatidylcholines are the largest population, making up 33-45\% of total lipid mass, and play critical roles in particle stability, cholesterol efflux, and molecular interactions with HDL-associated enzymes (10).

HDL can be classified into different subpopulations using various techniques. An overview of $\mathrm{HDL}$ classification, based on the publication by Rosenson et al (11), is shown in Table 1. Briefly, the use of density-gradient ultracentrifugation and nondenaturing gradient gel electrophoresis can distinguish HDL subpopulations on the basis of density and size, respectively; from smallest to largest: HDL3c, HDL3b, HDL3a, HDL2a, and HDL2b (11). Apo A-I containing HDL subpopulations can also be defined on the basis of size and charge: pre- $\beta-1 \mathrm{HDL}$ (very small, discoidal HDL with Apo A-I and phospholipid), a-4 HDL (small, discoidal HDL with Apo A-I, phospholipid, and free cholesterol), a-3 HDL (medium-sized, spherical HDL with Apo A-I, Apo A-II, phospholipid, free cholesterol, cholesteryl ester, and triglyceride), a-2 HDL (large, spherical HDL with the same constituents as a-3 HDL), and a-1 HDL (very large, spherical HDL with the same constituents as a-3 HDL but nearly no Apo A-II) (11). Using more sophisticated techniques, such as nuclear magnetic resonance (NMR), 26 different HDL subpopulations have been identified, but they are simply described as small, medium, and large due to limited measurement precision (11). Recently, an NMR-based clinical analyzer called Vantera was developed to measure total HDL particle number in clinical laboratory settings (12).

\section{Changes in HDL composition in SLE patients}

Dyslipoproteinemia. Many SLE patients have increased levels of very low-density lipoproteins and lowdensity lipoproteins (LDLs) and decreased levels of HDL, considered the "lupus lipoprotein pattern." Low HDL cholesterol is one of the most prevalent dyslipidemia indicators observed in SLE patients, including pediatric populations (13-18). In a multiethnic US cohort study containing 546 SLE patients, 81\% had low HDL cholesterol levels (<35 mg/dl) (17). In a recent Egyptian study, $45 \%$ of 221 SLE patients presented with low HDL cholesterol levels (<40 mg/dl) (18). In some instances, no significant differences in HDL cholesterol levels were found between SLE patients and controls, which may be explained by differences in the patient population (7). Nevertheless, other 
Table 1. Classification of $\mathrm{HDL}^{*}$

\begin{tabular}{|c|c|c|c|c|c|}
\hline Separation and analytical methods & Very small & Small & Medium & Large & Very large \\
\hline \multicolumn{6}{|l|}{ Density } \\
\hline Classification & - & HDL3 & - & HDL2 & - \\
\hline Range, $F_{1.2}$ & - & $0-3.5$ & - & $3.5-9$ & - \\
\hline \multicolumn{6}{|l|}{ Density-gradient ultracentrifugation } \\
\hline Classification & HDL3C & HDL3b & HDL3a & HDL2a & HDL2b \\
\hline Range, gm/ml & $1.15-1.17$ & $1.13-1.15$ & $1.11-1.13$ & $1.09-1.11$ & $1.06-1.09$ \\
\hline \multicolumn{6}{|l|}{ Size } \\
\hline \multicolumn{6}{|c|}{ Nondenaturing gradient gel electrophoresis } \\
\hline Classification & HDL3C & HDL3b & HDL3a & HDL2a & HDL2b \\
\hline Range, nm & $7.2-7.8$ & $7.8-8.2$ & $8.2-8.8$ & $8.8-9.7$ & $9.7-12.9$ \\
\hline \multicolumn{6}{|l|}{ 2-D gel electrophoresis } \\
\hline Classification & pre- $\beta-1$ & $a-4$ & $a-3$ & $a-2$ & $a-1$ \\
\hline Range, nm & $5.0-6.0$ & $7.0-7.5$ & $8.5-8.5$ & $9.0-9.4$ & $10.8-11.2$ \\
\hline \multicolumn{6}{|l|}{ NMR } \\
\hline Classification & & Small & Medium & Large & \\
\hline Range, nm & - & $7.3-8.2$ & $8.2-9.4$ & $9.4-14$ & - \\
\hline
\end{tabular}

* HDL = high-density lipoprotein; 2-D = 2-dimensional; NMR = nuclear magnetic resonance.

HDL-related abnormalities were identified in those studies, such as reduced paraoxonase 1 (PON-1) activity (7).

It is noteworthy that HDL cholesterol, which is routinely determined in clinical laboratories, is usually considered synonymous to HDL particle level. However, it has been increasingly recognized that HDL cholesterol may not be an appropriate surrogate of HDL levels, since the cholesterol content of HDL does not correlate perfectly with the number of HDL particles (19). Alternatively, some studies determined HDL levels by measuring Apo A-I concentrations using immunochemistry methods, and lower Apo A-I levels were found in SLE patients relative to healthy subjects (13). Recently, direct measurement of HDL particle numbers has been made possible in clinical laboratories using NMR methods (12). Chung et al found that SLE patients had lower numbers of large HDL particles compared to healthy volunteers when evaluated by NMR (20). Patients with active disease have lower HDL levels (21-24), while the use of prednisone and hydroxychloroquine correlates with higher HDL cholesterol $(23,25)$. In contrast, simvastatin and atorvastatin have not been found to modify HDL cholesterol levels in SLE patients $(26,27)$.

Changes in HDL size distribution in SLE patients. Lupus patients have been found to have a different distribution of HDL subfractions and HDL sizes compared to healthy controls (Table 2). However, the reported HDL changes vary between individual studies, likely due to the differences in patient inflammatory states as well as medications administered in each study to control SLE, both affecting HDL distribution. In

Table 2. Changes in lipoprotein profile and HDL composition in SLE patients*

\begin{tabular}{|c|c|c|}
\hline Author, year (ref.) & Subjects & HDL changes \\
\hline Delgado Alves et al, 2002 (35) & 32 lupus patients and 20 matched controls & $\begin{array}{l}\text { Decreased PON-1 activity } \\
\text { Decreased HDL2 } \\
\text { Decreased HDL3 }\end{array}$ \\
\hline McMahon et al, 2006 (38) & 154 women with SLE & Increased proinflammatory HDL \\
\hline Kiss et al, 2007 (7) & 37 SLE patients & $\begin{array}{l}\text { Decreased Apo A-I } \\
\text { Decreased PON-I activity }\end{array}$ \\
\hline Juárez-Rojas et al, 2008 (29) & $\begin{array}{l}30 \text { women with uncomplicated SLE and } \\
18 \text { matched controls }\end{array}$ & $\begin{array}{l}\text { Decreased cholesteryl ester } \\
\text { Increased triglycerides } \\
\text { Decreased Apo A-I } \\
\text { Decreased HDL size } \\
\text { Decreased HDL2b } \\
\text { Increased HDL3b and HDL3c }\end{array}$ \\
\hline Batuca et al, 2009 (34) & 77 lupus patients & Decreased PON-1 activity \\
\hline Smith et al, 2014 (39) & SLE patients and healthy controls & Increased oxidized Apo A-I \\
\hline Marsillach et al, 2015 (60) & 54 SLE patients and 25 matched healthy controls & Decreased PON-3 \\
\hline Gaál et al, 2016 (16) & 51 SLE patients and 49 matched healthy controls & $\begin{array}{l}\text { Increased SAA combination } \\
\text { Decreased PON-I arylesterase activity }\end{array}$ \\
\hline Han et al, 2016 (33) & 18 SLE patients & Increased SAA combination \\
\hline
\end{tabular}

* $\mathrm{HDL}$ = high-density lipoprotein; SLE = systemic lupus erythematosus; PON-1 = paraoxonase 1; Apo A-I = apolipoprotein A-l; SAA = serum amyloid $A$. 
addition, the analytical methodologies used to characterize HDL distribution differ between studies, further hindering comparison of the results. Hua et al reported that small HDL is less prevalent in SLE patients, while the overall HDL size is increased in SLE patients compared to healthy controls (28). Chung et al reported that SLE patients have a lesser proportion of large HDL; however, the overall sizes of HDL were no different between SLE and healthy individuals (20). Other studies have also reported no significant differences in HDL size between SLE patients and healthy controls (16). Juárez-Rojas et al reported that SLE patients tend to have lower proportions of HDL2b and higher proportions of HDL3b and HDL3c (29). Similar results were reported by Formiga et al, where SLE patients had higher levels of HDL3 cholesterol and lower levels of HDL2 cholesterol (30).

Proteomic and lipidomic changes in SLE HDL composition. HDL in SLE also has abnormal proteomic features, with changes in Apo A-I being the most noteworthy. In the study by Machado et al, female adolescents with SLE had a higher ratio of HDL cholesterol to Apo A-I compared to healthy controls, indicating that the HDL particles in those SLE patients had a lesser amount of Apo A-I (31). JuárezRojas et al reported lower Apo A-I and higher Apo E content in HDL particles isolated from women with SLE (29). However, in another study comparing 51 SLE patients to 49 healthy controls, SLE patients were found to have a higher ratio of Apo A-I to HDL, although the overall serum Apo A-I level was lower (16). Similar to proteomic alterations, the lipidome of HDL is also compromised in the SLE setting. Juárez-Rojas et al found that HDL isolated from SLE patients had less cholesteryl ester and more triglycerides compared to healthy controls (29). Moreover, lipid peroxidation is enhanced in SLE patients due to enhanced oxidative stress that promotes a significant increase in the production of oxidized lipids (32). Further, Gaál et al (16) and Han et al (33) found that SLE patients display significantly increased levels of serum amyloid A (SAA) compared to healthy subjects. Additionally, multiple studies confirmed a significant reduction in PON-1 and PON-3 activity in SLE patients $(7,16,34,35)$. The abnormal lipoprotein profiles in SLE patients are summarized in Table 2.
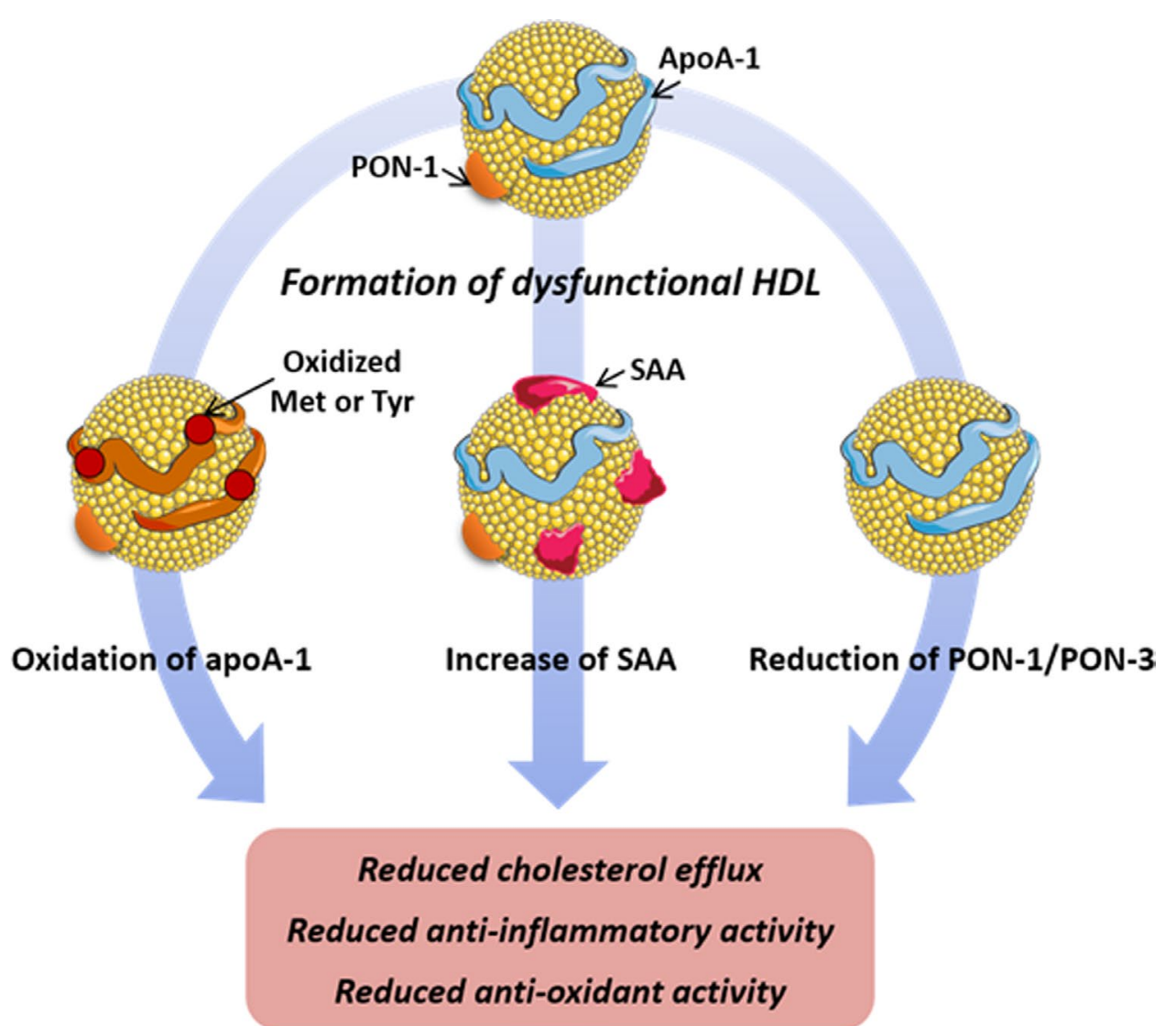

Figure 2. Formation of dysfunctional or proinflammatory high-density lipoprotein (HDL) in systemic lupus erythematosus (SLE). In SLE, multiple HDL proteomic changes occur, leading to the impairment of the function of HDL. Oxidation of apolipoprotein A-I (Apo A-I) methionine (Met) and tyrosine (Tyr) residues by chemical and enzymatic pathways leads to reduced ability of HDL to efflux cholesterol outside the cell and neutralize oxidized lipids. Increased levels of serum amyloid A (SAA) cause displacement of Apo A-I on HDL and reduced cholesterol efflux and antiinflammatory activity. Reduced levels and activity of HDL-associated paraoxonase 1 (PON-1) lead to the reduced antioxidant activity of HDL. 
Proinflammatory HDL. HDL exerts antiinflammatory functions in healthy subjects. However, in the course of inflammatory disease such as SLE, the composition of HDL is altered and its function compromised. This can lead to the transformation of HDL into a dysfunctional, proinflammatory particle with reduced cholesterol efflux capacity unable to perform its normal antiinflammatory and antioxidative functions (Figure 2) (36). Compared to normal HDL, proinflammatory HDL is characterized by increased SAA content, decreased Apo A-I levels, increased Apo A-I oxidation, and decreased PON activity (16). Increased SAA content in HDL results in its displacement of Apo A-I, thus, reducing the atheroprotective properties of HDL (37). McMahon et al reported that $44.7 \%$ of SLE patients had increased levels of proinflammatory HDL in comparison to only $4.1 \%$ of controls and $20.1 \%$ of rheumatoid arthritis patients (38). Their subsequent study identified proinflammatory HDL to be associated with increased carotid intima-media thickness and plaque by carotid ultrasound, suggesting that dysfunctional proinflammatory HDL significantly contributes to the development of subclinical atherosclerosis in SLE (6).

Oxidized HDL. HDL also undergoes structural changes in SLE due to oxidation, and it has been found that SLE patients have elevated levels of oxidized HDL (Figure 2) (39). Myeloperoxidase (MPO) is the main oxidant enzyme responsible for the oxidation of HDL. MPO-catalyzed oxidation converts normal tyrosine on Apo A-I to 3-chlorotyrosine or 3-nitrotyrosine, leading to oxidized HDL (40). MPO also oxidizes 3 methionine residues of Apo A-I, methionine 86 (Met-86), Met-112, and Met-148, contributing significantly to enhancing levels of oxidized Apo A-I (41). In SLE patients, a high concentration of serum MPO is reported, implying a link between increased HDL oxidation and increased MPO levels in this disease. Recent studies found associations between HDL oxidation and elevated formation of neutrophil extracellular traps (NETs) (39). Under normal conditions, NETs play an important antimicrobial role; however, in SLE, NETs are dysregulated. It has been shown that the degradation of NETs is hindered in SLE patients, resulting in aberrant elevation of NETs and infiltration of netting neutrophils in tissues (42).

Carlucci et al demonstrated that SLE patients present higher levels of low-density granulocytes (LDGs), which are one subset of neutrophils with enhanced NET formation capacity (43). The increased LDG levels are found to be associated with the impaired cholesterol efflux capacity of HDL isolated from lupus patients (43). It has been proposed that enhanced levels of NETs enhances the oxidant potential in SLE and leads to the externalization to the extracellular space of oxidant enzymes such as MPO, nitric oxide synthase (NOS), and NADPH oxidase (NOX), ultimately promoting oxidation of HDL (Figure 3) $(43,44)$. Data from SLE patients showed that MPO and NOX from NETs promoted 3-chlorotyrosine modification, and NOS and NOX promoted 3-nitrotyrosine modification on HDL, while inhibition of NETs decreased the oxidation of HDL. These observations support the notion that NETs play a major role in the oxidation of HDL in SLE (39).

In addition to MPO, lipid peroxidation products also mediate the oxidation of HDL. Studies showed that oxidized phospholipids could covalently react with Apo A-I at various sites, forming lipid-protein adducts (45). It was also found that lipid hydroperoxide could chemically react with Apo A-I and Apo A-II, leading to the oxidation of Met residues to methionine sulfoxide (46). Since clinical studies have shown that lipid peroxidation is enhanced in SLE patients, this may also contribute to the increased oxidation of Apo A-I and HDL in this disease (47).

\section{Functional changes in HDL in SLE patients}

Impaired cholesterol efflux capacity. Removal of excess cholesterol from macrophages in the artery wall is recognized to be the key process of HDL for protection against atherosclerosis and improvement of cardiovascular outcomes. This process, known as reverse cholesterol transport, allows translocating excess cholesterol and other lipids from lipid-laden macrophages in atherosclerotic lesions to the liver for elimination. The first and most crucial step in reverse cholesterol transport is to efflux cholesterol from macrophages to HDL. HDL isolated from SLE patients displays a 15\% decrease in cholesterol efflux ability compared to healthy control HDL (39). The decreased cholesterol efflux capacity of HDL purified from SLE patients has been found to significantly correlate with increased noncalcified coronary plaque burden (43). Multiple studies have proposed that the reason for decreased cholesterol efflux ability of HDL in SLE is increased levels of SAA. As mentioned earlier, increased levels of SAA promote dysfunctional proinflammatory HDL with diminished ability to remove cholesterol from macrophages and traffic cholesteryl ester to the liver (48).

Oxidation of HDL can also contribute to its reduced cholesterol efflux capacity in SLE. HDL containing oxidized Apo A-I has a reduced ability to efflux cholesterol $(49,50)$. Furthermore, when Met-148 is oxidized in Apo A-I, HDL loses the ability to interact with lecithin-cholesterol acyltransferase, an enzyme responsible for the conversion to cholesterol ester, which is a key step in reverse cholesterol transport (51). Thus, the oxidation of Apo A-I could be a key reason for the impaired process of cholesterol efflux in SLE.

Reduced antiinflammatory function. In recent years, it has become widely accepted that HDL can directly inhibit the inflammation processes that lead to the development of atherosclerosis (52). Although the complex antiinflammatory mechanisms of HDL have not been fully elucidated, it has been suggested that several signaling pathways play a role in this process. First, HDL can inhibit Toll-like receptor (TLR) pathways through activation of the transcriptional repressor activating transcription factor 3 (ATF3). Activated ATF-3 is translocated into the nucleus and inhibits 


\section{Formation of dysfunctional HDL in SLE}

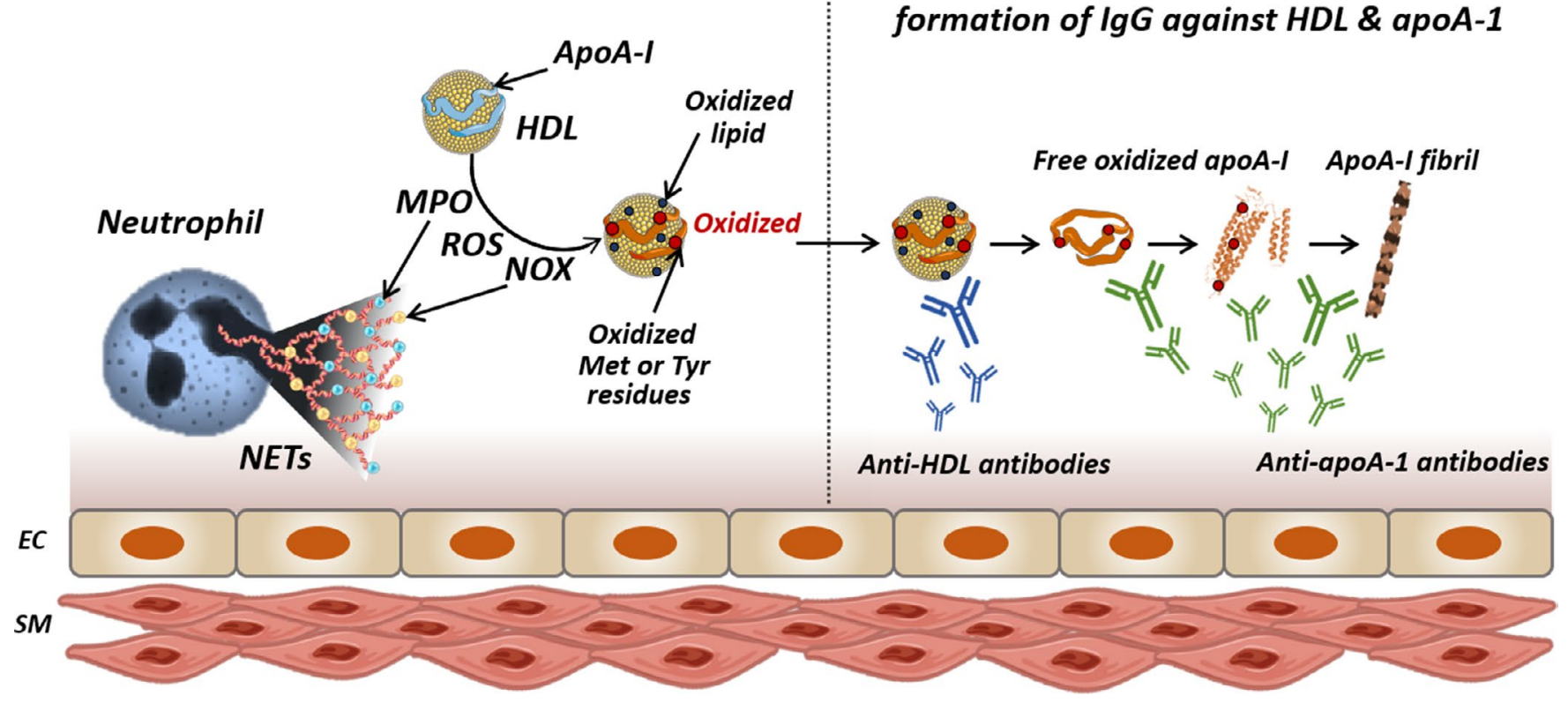

Figure 3. Formation of dysfunctional high-density lipoprotein (HDL) and autoantibodies against HDL and apolipoprotein A-I (Apo A-I) in systemic lupus erythematosus (SLE). In SLE, abnormal elevation of neutrophil extracellular traps (NETs) is observed, leading to endothelial cell (EC) damage. In the presence of NETs, increased levels of myeloperoxidase (MPO), nitric oxide synthase (NOS), NADPH oxidase (NOX), and reactive oxygen species (ROS) are observed, causing oxidation of HDL and Apo A-I. Oxidation of Apo A-I and HDL induces the formation of anti-HDL and anti-Apo A-I autoantibodies. Furthermore, oxidation of Apo A-I at Met-148 leads to conformational changes in Apo A-I, promoting protein misfolding, dissociation of misfolded Apo A-I from HDL, and formation of Apo A-I amyloid fibrils. This aggregated Apo A-I is more immunogenic, leading to a further increase in anti-Apo A-I autoantibody titers. SM = smooth muscle cell.

the promotion of TLR-induced inflammatory cytokines (53). HDL can also inhibit NF-kB-activated cell adhesion molecule expression, thus preventing vascular inflammation (54).

In contrast to the antiinflammatory response promoted by healthy HDL, HDL purified from SLE patients induces a proinflammatory response. Smith et al reported that HDL in SLE fails to inhibit cytokine induction driven by TLR pathways (55). In comparison to macrophages exposed to healthy HDL, macrophages treated with SLE HDL induced activation of NF-KB and increased expression of tumor necrosis factor (TNF) and interleukin-6 (IL6). In addition, macrophages treated with SLE HDL had significantly repressed ATF-3 activation compared to control HDL or an untreated group, suggesting that HDL from SLE patients cannot inhibit TLR pathways via ATF-3 activation.

The oxidation of HDL in SLE promotes the binding of HDL to the lectin-like oxidized low-density lipoprotein receptor 1 , preventing ATF-3 nuclear translocation and leading to increased synthesis of inflammatory cytokines (55). HDL from SLE patients was also found to be able to directly up-regulate monocyte plateletderived growth factor receptor $\beta$ and increase chemotaxis and TNF release (56). Another study associated the loss of antiinflammatory function of HDL in SLE with the increased SAA content in HDL particles. Under normal conditions, HDL can inhibit inflammatory responses by disrupting lipid rafts and sequestering plasma membrane cholesterol; however, it has been suggested that the SAA binding on the surface of HDL impedes the interaction between HDL and cell membrane, decreasing the antiinflammatory role of $\mathrm{HDL}$ (33).

Reduced antioxidant capacity. In both the general population and in individuals with SLE, increased oxidized LDL level is a well-recognized risk factor for CVD (57). Under normal conditions, HDL prevents LDL oxidation by scavenging reactive oxygen species (ROS). The increased levels of oxidized HDL and oxidized Apo A-l in SLE reduce the ability of HDL particles to scavenge ROS. In addition, proinflammatory HDL in SLE may promote LDL oxidation. Elevated levels of oxidized LDL increase recruitment and adherence of monocytes to activated endothelial cells by increasing the expression of adhesion molecules and proinflammatory cytokines (58). These monocytes then transmigrate to the arterial intima, taking up oxidized LDL and eventually maturing to form foam cells.

PON enzymes also play a critical role in the antioxidant functions of $\mathrm{HDL}$. Among the PON enzymes, PON-1 is the major antioxidant in $\mathrm{HDL}$ and prevents $\mathrm{LDL}$ from oxidation, thereby eliminating biologically active oxidized LDL (46). SLE patients have reduced PON-1 activity $(7,16,34,35,59)$ and this correlates with the loss in antioxidative function of HDL (16). While the reasons for the loss in PON-1 activity are not fully understood, levels of 
various autoantibodies inversely correlate with $\mathrm{PON}-1$ activity. These include IgG against Apo A-I (8,16,34), HDL (34,35), and $\beta_{2}$-glycoprotein I (35), suggesting that autoantibodies may contribute to the decreased activity of $\mathrm{PON}-1$. In addition to $\mathrm{PON}-1$, $\mathrm{PON}-3$, another member of the PON enzyme family, is decreased in SLE patients with subclinical atherosclerosis, potentially promoting the loss of the antioxidant ability of HDL in SLE (60) (Figure 2).

\section{Formation of autoantibodies in SLE}

The production of autoantibodies is the key manifestation of SLE. In patients affected by autoimmune disorders, highly reactive IgG antibodies against human Apo A-I are detected and can bind to both lipid-free Apo A-I as well as Apo A-I on HDL particles (34). It has been reported that $32.5 \%$ of patients with SLE tested positively for the presence of anti-Apo A-I autoantibodies in association with decreased levels of HDL (61). Similar studies have confirmed the presence of anti-Apo A-I and its association with higher disease activity in SLE patients $(34,62,63)$. In comparison, only $1 \%$ of healthy individuals and $20 \%$ of patients with acute coronary syndrome without an autoimmune disorder have detectable levels of anti-Apo A-I (64). The presence of anti-Apo A-I has been reported to reduce the activity of $\mathrm{PON}$, leading to increased LDL oxidation $(34,35)$. Likewise, in lupus-prone murine models, antiApo A-I was associated with decreased levels of HDL cholesterol and $\mathrm{PON}-1$ activity (8).

We hypothesize that increased levels of anti-Apo A-I in SLE patients correlates with increased lipid-free Apo A-I and oxidized free Apo A-I. Lipid-free Apo A-I can exist in plasma via several pathways, either due to de novo synthesis or dissociation from HDL due to displacement by elevated levels of SAA. In addition, oxidation of Apo A-I at the Met-148 position leads to conformational changes in the protein (65). Oxidation of Apo A-I favors protein misfolding from the native a-helical structure to $\beta$-sheets, facilitating dissociation of Apo A-I from HDL particles and initiating Apo A-I amyloid fibril formation (41). Thus, in SLE plasma and in the oxidative microenvironment associated with NETs and atherosclerosis, the levels of structurally modified Apo A-I are likely to be elevated, leading to higher levels of lipid-free misfolded protein (Figure 3). Indeed, in HDL isolated from SLE patients, the median levels of 3-nitrotyrosine and 3-chlorotyrosine were 1.9- and 120.9fold higher than in HDL isolated from healthy controls (39). The misfolded and oxidized Apo A-I protein is likely more immunogenic, thus leading to higher titers of anti-Apo A-I in SLE.

Anti-HDL antibodies have recently been identified in SLE patients. The differences between anti-HDL antibodies and anti-Apo A-I antibodies remain unclear $(34,61)$. Lipid-free Apo A-I-coated enzyme-linked immunosorbent assay is most commonly used to measure antibody titers in serum. However, in some instances, the entire HDL particle is used in the assay. It is possible that HDL used in the assay is partially oxidized and antibodies present in SLE patient serum recognize either oxidized or partially misfolded Apo A-I that remains to be lipid-bound in HDL particles (66). A few studies have reported significantly elevated levels of anti-HDL in SLE compared to either healthy subjects or patients with primary antiphospholipid syndrome $(67,68)$. High anti-HDL titers were associated with increased SLE disease activity markers and decreased PON activity, which could lead to loss of antioxidant and atheroprotective functions of HDL and promotion of atherosclerosis development $(7,34,60)$.

\section{Applications of HDL therapeutics}

The impact of statin use to control CVD development in SLE patients has been examined in several clinical studies. The Lupus Atherosclerosis Prevention Study showed no significant difference in carotid intima-media thickness of carotid plaque between SLE patients treated with atorvastatin and those treated with placebo (27). However, a recent nationwide population-based cohort study utilizing statin therapy showed promising results, with a significant reduction of risk of CVD mortality by $30 \%$ in SLE patients with hyperlipidemia (69). In addition, short-term atorvastatin therapy improved endothelium-dependent vasodilation in SLE patients (70). Therefore, sufficiently powered long-term prospective clinical trials are necessary to definitively conclude whether the use of statins is beneficial in SLE patients.

Reconstituted HDL, nanoparticles prepared from Apo A-I or Apo A-I mimetic peptides following reconstitution with phospholipids, have been extensively studied as an antiatherosclerosis therapeutic since 1984 (71). Infusions of reconstituted HDL have been shown to increase levels of circulating HDL, improve plasma cholesterol efflux capacity, inhibit the synthesis of proinflammatory mediators, and improve endothelial function, leading to increased overall atheroprotection in animal models and in clinical trials (72). As of today, 6 different reconstituted HDL products have been tested in clinical trials, including SRC-rHDL, CSL-111, ETC-216, ETC-642, CER-001, and CSL-112. Two products, ETC-216 and CSL-111, were shown to reduce plaque burden in CVD patients as assessed by intravascular ultrasound $(73,74)$. CSL-112, a newer formulation of CSL-111, exhibited improved clinical safety and cholesterol efflux capacity in healthy volunteers compared to CSL-111 (75). A phase III study of 17,400 patients to explore the ability of CSL-112 to reduce major adverse cardiovascular events in CVD patients is currently ongoing (76).

HDL therapy has also presented beneficial effects on inflammatory disorders such as sepsis. It can neutralize endotoxin from bacteria, regulate the inflammatory response in macrophages, and inhibit endothelial cell activation in sepsis (77). As a result, the infusion of CSL-111 suppressed proinflammatory cytokine production, sepsis-induced hypotension, and reduced the severity of clinical symptoms (78). Similarly, L-4F, an Apo A-I mimetic peptide, demonstrated a beneficial effect in studies of various animal sepsis models by inhibiting proinflammatory cytokine production, reducing sepsis-induced hypotension, protecting 


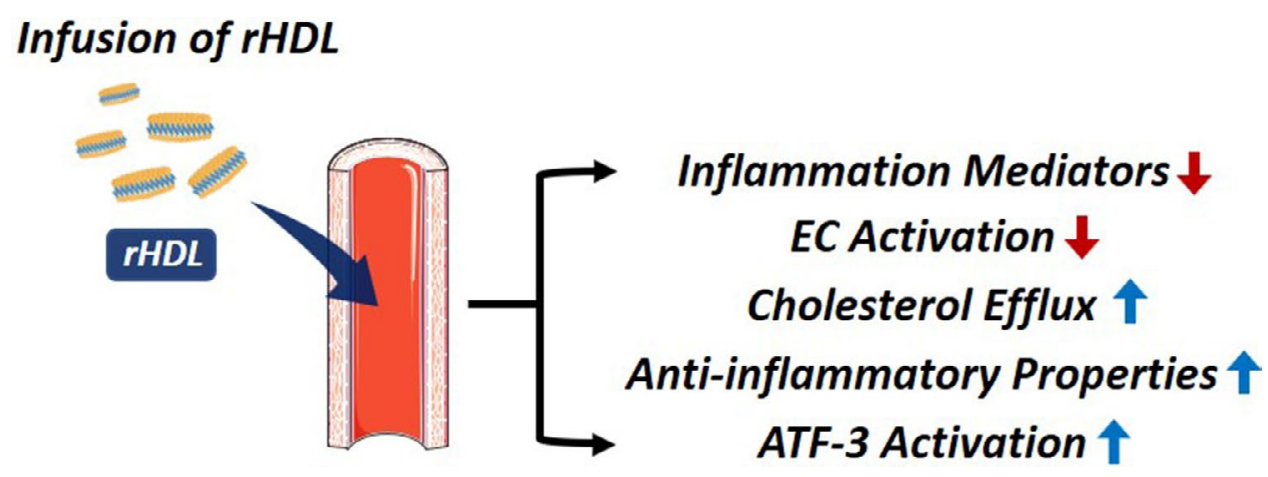

Figure 4. Reconstituted HDL (rHDL) as a putative therapeutic strategy in patients with systemic lupus erythematosus (SLE) at risk of cardiovascular disease (CVD). Infusion of reconstituted HDL in SLE patients may increase the level of pre- $\beta$-HDL, reduce the presence of inflammatory mediators and activation of endothelial cells, enhance antiinflammatory properties and activating transcription factor 3 (ATF-3) activation, and facilitate cholesterol efflux capacity.

against organ damage, and increasing the survival rate $(79,80)$. Charles-Schoeman et al have proposed a possible therapy with Apo A-I mimetic peptides in collagen-induced arthritis, a rodent model of rheumatoid arthritis (81). Rats treated with combination therapy of Apo A-I mimetic peptides, D-4F, and pravastatin had a significantly improved clinical severity score and less erosive disease compared to both rats that received noncombination treatment and control groups. Levels of inflammatory cytokines and chemokines were notably reduced with combination therapy and the antiinflammatory properties of HDL were improved.

Use of Apo A-I mimetic peptides and reconstituted HDL in animal models of SLE. To date, very few studies have tested the putative benefit of HDL treatment in animal models of SLE. A study by Woo et al showed significant improvements in SLE manifestations in a murine lupus model associated with accelerated atherosclerosis via treatment with the Apo A-I mimetic peptide, L-4F, alone or with pravastatin (82). Notably, treatment with L-4F alone or with pravastatin significantly reduced IgG anti-double-stranded DNA (anti-dsDNA), IgG antioxidized phospholipids, proteinuria, glomerulonephritis, and osteopenia. L-4F also improved the antiinflammatory functions of plasma HDL while reducing the proinflammatory effects of LDL. In a more recent study by Black et al, increased levels of Apo A-I resulted in suppression of lymphocyte activation, IgG anti-dsDNA autoantibodies, interferon- $y$-secreting CD4+ Th1 cells, and follicular T helper cells, along with improved glomerulonephritis in a normocholesterolemic murine model of SLE (83). Smith et al investigated whether reconstituted $\mathrm{HDL}$ can reverse the proinflammatory effects of lupus HDL by administering ETC-642, an HDL mimetic composed of the Apo A-I mimetic peptide (ESP24218) and phospholipid complex, in vivo to NZM2328 mice, a mouse model of lupus (55). Indeed, macrophages exposed to a 1:4 ratio of SLE HDL to ETC-642 significantly suppressed TNF, IL-6, and NF-kB activation while promoting ATF-3 nuclear translocation, suggesting that reconstituted $\mathrm{HDL}$ can successfully mimic the effects of healthy HDL. The therapy led to significant increases in ATF-3 expression and lowered IL-6 levels in serum, indicating that reconsituted HDL can also decrease cytokine synthesis.

Future directions for reconstituted HDL therapy in SLE patients with CVD. Underscoring the fact that dysfunctional HDL may promote CVD in SLE, HDL therapy may serve as a potential and alternative therapeutic because it can restore both the quantity and quality of HDL. It is worth noting that reconstituted HDL and naked Apo A-I mimetic peptides used in previous studies have not been optimized for CVD prevention and treatment trials in SLE, as they primarily focused on maximizing the efficacy of cholesterol efflux. Therefore, the potential benefit of reconstituted HDL therapy for CVD prevention and treatment in SLE patients remains unclear. An advantage of reconstituted HDL therapy is that it can be further customized to be more disease specific. We have recently discovered that the lipid component of HDL can significantly alter the cholesterol efflux capacity and antiinflammatory properties of HDL (84). Therefore, further investigations to understand the protective roles of reconstituted HDL in SLE with CVD are needed and will inform the optimization of the reconstituted HDL composition. Administration of optimized reconstituted HDL in SLE models would likely increase the level of pre- $\beta-\mathrm{HDL}$ and may restore the antiinflammatory functions of HDL, and reduce proinflammatory HDL and oxidized LDL. Improved and restored HDL may then exert its diverse protective mechanisms, including the reduction of inflammatory mediators and activation of endothelial cells, all while improving cholesterol efflux capacity (Figure 4). In addition, prevention of autoantibody recognition may be possible in reconstituted HDL therapy via altering protein/peptide composition. These observations suggest that HDL mimetics may serve as an effective therapeutic strategy for reducing CVD risk and, potentially, disease activity in SLE.

\section{AUTHOR CONTRIBUTIONS}

All authors drafted the article, revised it critically for important intellectual content, and approved the final version to be published. 


\section{REFERENCES}

1. Urowitz MB, Gladman DD, Tom BD, Ibañez D, Farewell VT. Changing patterns in mortality and disease outcomes for patients with systemic lupus erythematosus. J Rheumatol 2008;35:2152-8.

2. Bernatsky S, Boivin JF, Joseph L, Manzi S, Ginzler E, Gladman DD, et al. Mortality in systemic lupus erythematosus. Arthritis Rheum 2006;54:2550-7.

3. Agarwal S, Elliott JR, Manzi S. Atherosclerosis risk factors in systemic lupus erythematosus. Curr Rheumatol Rep 2009;11:241-7.

4. Lerang K, Gilboe IM, Steinar Thelle D, Gran JT. Mortality and years of potential life loss in systemic lupus erythematosus: a populationbased cohort study. Lupus 2014;23:1546-52.

5. Besler C, Lüscher TF, Landmesser U. Molecular mechanisms of vascular effects of high-density lipoprotein: alterations in cardiovascular disease. EMBO Mol Med 2012;4:251-68.

6. McMahon M, Grossman J, Skaggs B, FitzGerald J, Sahakian L, Ragavendra N, et al. Dysfunctional proinflammatory high-density lipoproteins confer increased risk of atherosclerosis in women with systemic lupus erythematosus. Arthritis Rheum 2009;60:2428-37.

7. Kiss E, Seres I, Tarr T, Kocsis Z, Szegedi G, Paragh G. Reduced paraoxonase1 activity is a risk for atherosclerosis in patients with systemic lupus erythematosus. Ann N Y Acad Sci 2007;1108:83-91.

8. Srivastava R, Yu S, Parks BW, Black LL, Kabarowski JH. Autoimmune-mediated reduction of high-density lipoproteincholesterol and paraoxonase 1 activity in systemic lupus erythematosus-prone gld mice. Arthritis Rheum 2011;63:201-11.

9. Kontush A, Lindahl M, Lhomme M, Calabresi L, Chapman MJ, Davidson WS. Structure of HDL: particle subclasses and molecular components. Handb Exp Pharmacol 2015;224:3-51.

10. Kontush A, Lhomme M, Chapman MJ. Unraveling the complexities of the HDL lipidome. J Lipid Res 2013;54:2950-63.

11. Rosenson RS, Brewer HB, Chapman MJ, Fazio S, Hussain MM, Kontush A, et al. HDL measures, particle heterogeneity, proposed nomenclature, and relation to atherosclerotic cardiovascular events. Clin Chem 2011;57:392-410.

12. Matyus SP, Braun PJ, Wolak-Dinsmore J, Saenger AK, Jeyarajah EJ, Shalaurova I, et al. HDL particle number measured on the Vantera ${ }^{\circledR}$, the first clinical NMR analyzer. Clin Biochem 2015;48:148-55.

13. Lilleby V, Haugen M, Mørkrid L, Frøslie FK, Holven KB, Førre Ø. Body composition, lipid and lipoprotein levels in childhood-onset systemic lupus erythematosus. Scand J Rheumatol 2007;36:40-7.

14. Soep JB, Mietus-Snyder M, Malloy MJ, Witztum JL, von Scheven E. Assessment of atherosclerotic risk factors and endothelial function in children and young adults with pediatric-onset systemic lupus erythematosus. Arthritis Rheum 2004;51:451-7.

15. Yuan J, Li LI, Wang Z, Song W, Zhang Z. Dyslipidemia in patients with systemic lupus erythematosus: association with disease activity and B-type natriuretic peptide levels. Biomed Rep 2016;4:68-72.

16. Gaál K, Tarr T, Lőrincz H, Borbás V, Seres I, Harangi M, et al. Highdensity lipopoprotein antioxidant capacity, subpopulation distribution and paraoxonase-1 activity in patients with systemic lupus erythematosus. Lipids Health Dis 2016;15:60.

17. Toloza SM, Uribe AG, McGwin G Jr, Alarcón GS, Fessler BJ, Bastian $\mathrm{HM}$, et al. Systemic lupus erythematosus in a multiethnic US cohort (LUMINA). XXIII. Baseline predictors of vascular events. Arthritis Rheum 2004;3947-57.

18. Gamal SM, Fawzy SM, Abdo M, Elgengehy FT, Ghoniem S, Alkemry A. Immunological profile and dyslipidemia in Egyptian systemic lupus erythematosus patients. Egypt Rheumatol 2017;39:89-92.

19. Heinecke JW. The not-so-simple HDL story: a new era for quantifying HDL and cardiovascular risk? Nat Med 2012;18:1346-7.
20. Chung CP, Oeser A, Raggi P, Solus JF, Avalos I, Linton MF, et al. Lipoprotein subclasses and particle size determined by nuclear magnetic resonance spectroscopy in systemic lupus erythematosus. Clin Rheumatol 2008;27:1227-33.

21. Wijaya LK, Kasjmir YI, Sukmana N, Subekti I, Prihartono J. The proportion of dyslipidemia in systemic lupus erythematosus patient and distribution of correlated factors. Acta Med Indones 2005;37:132-44.

22. Kashef S, Ghaedian MM, Rajaee A, Ghaderi A. Dyslipoproteinemia during the active course of systemic lupus erythematosus in association with anti-double-stranded DNA (anti-dsDNA) antibodies. Rheumatol Int 2007;27:235-41.

23. Sarkissian T, Beyenne J, Feldman B, Adeli K, Silverman E. The complex nature of the interaction between disease activity and therapy on the lipid profile in patients with pediatric systemic lupus erythematosus. Arthritis Rheum 2006;54:1283-90.

24. Cardoso CR, Signorelli FV, Papi JA, Salles GF. Prevalence and factors associated with dyslipoproteinemias in Brazilian systemic lupus erythematosus patients. Rheumatol Int 2008;28:323-7.

25. Durcan L, Winegar DA, Connelly MA, Otvos JD, Magder LS, Petri M. Longitudinal evaluation of lipoprotein variables in systemic lupus erythematosus reveals adverse changes with disease activity and prednisone and more favorable profiles with hydroxychloroquine therapy. J Rheumatol 2016;43:745-50.

26. Schanberg LE, Sandborg C, Barnhart HX, Ardoin SP, Yow E, Evans GW, et al. Use of atorvastatin in systemic lupus erythematosus in children and adolescents. Arthritis Rheum 2012;64:285-96.

27. Petri MA, Kiani AN, Post W, Christopher-Stine L, Magder LS. Lupus Atherosclerosis Prevention Study (LAPS). Ann Rheum Dis 2011;70:760-5.

28. Hua X, Su J, Svenungsson E, Hurt-Camejo E, Jensen-Urstad K, Angelin B, et al. Dyslipidaemia and lipoprotein pattern in systemic lupus erythematosus (SLE) and SLE-related cardiovascular disease. Scand J Rheumatol 2009;38:184-9.

29. Juárez-Rojas J, Medina-Urrutia A, Posadas-Sánchez R, Jorge-Galarza E, Mendoza-Pérez E, Caracas-Portilla N, et al. High-density lipoproteins are abnormal in young women with uncomplicated systemic lupus erythematosus. Lupus 2008;17:981-7.

30. Formiga F, Meco JF, Pinto X, Jacob J, Moga I, Pujol R. Lipid and lipoprotein levels in premenopausal systemic lupus erythematosus patients. Lupus 2001;10:359-63.

31. Machado D, Sarni RO, Abad TT, Silva SG, Khazaal EJ, Hix S, et al. Lipid profile among girls with systemic lupus erythematosus. Rheumatol Int 2017;37:43-8.

32. Shah D, Mahajan N, Sah S, Nath SK, Paudyal B. Oxidative stress and its biomarkers in systemic lupus erythematosus [review]. J Biomed Sci 2014;21:23.

33. Han CY, Tang C, Guevara ME, Wei H, Wietecha T, Shao B, et al. Serum amyloid $A$ impairs the antiinflammatory properties of $\mathrm{HDL}$. $\mathrm{J}$ Clin Invest 2016;126:266-81.

34. Batuca JR, Ames PR, Amaral M, Favas C, Isenberg DA, Delgado Alves J. Anti-atherogenic and anti-inflammatory properties of high-density lipoprotein are affected by specific antibodies in systemic lupus erythematosus. Rheumatology (Oxford) 2009;48:26-31.

35. Delgado Alves J, Ames PR, Donohue S, Stanyer L, Nourooz-Zadeh $\mathrm{J}$, Ravirajan C, et al. Antibodies to high-density lipoprotein and $\beta_{2}$-glycoprotein I are inversely correlated with paraoxonase activity in systemic lupus erythematosus and primary antiphospholipid syndrome. Arthritis Rheum 2002;46:2686-94.

36. Hb G, Rao VS, Kakkar W. Friend turns foe: transformation of antiinflammatory HDL to proinflammatory HDL during acute-phase response. Cholesterol 2011;2011:274629.

37. Van Lenten BJ, Wagner AC, Nayak DP, Hama S, Navab M, Fogelman AM. High-density lipoprotein loses its anti-inflammatory properties during acute influenza A infection. Circulation 2001;103:2283-8. 
38. McMahon M, Grossman J, FitzGerald J, Dahlin-Lee E, Wallace DJ, Thong BY, et al. Proinflammatory high-density lipoprotein as a biomarker for atherosclerosis in patients with systemic lupus erythematosus and rheumatoid arthritis. Arthritis Rheum 2006;54:2541-9.

39. Smith CK, Vivekanandan-Giri A, Tang C, Knight JS, Mathew A, Padilla $R L$, et al. Neutrophil extracellular trap-derived enzymes oxidize highdensity lipoprotein: an additional proatherogenic mechanism in systemic lupus erythematosus. Arthritis Rheumatol 2014;66:2532-44.

40. Heinecke JW. The role of myeloperoxidase in HDL oxidation and atherogenesis. Curr Atheroscler Rep 2007;9:249-51.

41. Chan GK, Witkowski A, Gantz DL, Zhang TO, Zanni MT, Jayaraman $\mathrm{S}$, et al. Myeloperoxidase-mediated methionine oxidation promotes an amyloidogenic outcome for apolipoprotein A-I. J Biol Chem 2015;290:10958-71.

42. Leffler J, Martin M, Gullstrand B, Tydén H, Lood C, Truedsson L, et al. Neutrophil extracellular traps that are not degraded in systemic lupus erythematosus activate complement exacerbating the disease. J Immunol 2012;188:3522-31.

43. Carlucci PM, Purmalek MM, Dey AK, Temesgen-Oyelakin $Y$, Sakhardande S, Joshi AA, et al. Neutrophil subsets and their gene signature associate with vascular inflammation and coronary atherosclerosis in lupus. JCI Insight 2018;3:99276.

44. Vivekanandan-Giri A, Slocum JL, Byun J, Tang C, Sands RL, Gillespie BW, et al. High density lipoprotein is targeted for oxidation by myeloperoxidase in rheumatoid arthritis. Ann Rheum Dis 2013;72:1725-31.

45. Szapacs ME, Kim HY, Porter NA, Liebler DC. Identification of proteins adducted by lipid peroxidation products in plasma and modifications of apolipoprotein A1 with a novel biotinylated phospholipid probe. J Proteome Res 2008;7:4237-46.

46. Hahn BH, Grossman J, Ansell BJ, Skaggs BJ, McMahon M. Altered lipoprotein metabolism in chronic inflammatory states: proinflammatory high-density lipoprotein and accelerated atherosclerosis in systemic lupus erythematosus and rheumatoid arthritis [review]. Arthritis Res Ther 2008;10:213.

47. Jiang $X$, Chen F. The effect of lipid peroxides and superoxide dismutase on systemic lupus erythematosus: a preliminary study. Clin Immunol Immunopathol 1992;63:39-44.

48. Artl A, Marsche G, Pussinen P, Knipping G, Sattler W, Malle E. Impaired capacity of acute-phase high density lipoprotein particles to deliver cholesteryl ester to the human $\mathrm{HUH}-7$ hepatoma cell line. Int J Biochem Cell Biol 2002;34:370-81.

49. Bergt C, Pennathur S, Fu X, Byun J, O’Brien K, McDonald TO, et al. The myeloperoxidase product hypochlorous acid oxidizes HDL in the human artery wall and impairs ABCA1-dependent cholesterol transport. Proc Natl Acad Sci U S A 2004;101:13032-7.

50. Shao B, Oda MN, Bergt C, Fu X, Green PS, Brot N, et al. Myeloperoxidase impairs ABCA1-dependent cholesterol efflux through methionine oxidation and site-specific tyrosine chlorination of apolipoprotein A-I. J Biol Chem 2006;281:9001-4.

51. Shao B, Cavigiolio G, Brot N, Oda MN, Heinecke JW. Methionine oxidation impairs reverse cholesterol transport by apolipoprotein A-I. Proc Natl Acad Sci U S A 2008;105:12224-9.

52. Säemann MD, Poglitsch M, Kopecky C, Haidinger M, Hörl WH, Weichhart T. The versatility of HDL: a crucial anti-inflammatory regulator. Eur J Clin Invest 2010;40:1131-43.

53. De Nardo D, Labzin LI, Kono H, Seki R, Schmidt SV, Beyer M, et al. High-density lipoprotein mediates anti-inflammatory reprogramming of macrophages via the transcriptional regulator ATF3. Nat Immunol 2014;15:152-60.

54. Park SH, Park JH, Kang JS, Kang YH. Involvement of transcription factors in plasma HDL protection against TNF-a-induced vascular cell adhesion molecule-1 expression. Int $\mathrm{J}$ Biochem Cell Biol 2003;35:168-82.

55. Smith CK, Seto NL, Vivekanandan-Giri A, Yuan W, Playford MP, Manna Z, et al. Lupus high-density lipoprotein induces proinflammatory responses in macrophages by binding lectin-like oxidised low-density lipoprotein receptor 1 and failing to promote activating transcription factor 3 activity. Ann Rheum Dis 2017;76:602-11.

56. Skaggs BJ, Hahn BH, Sahakian L, Grossman J, McMahon M. Dysfunctional, pro-inflammatory HDL directly upregulates monocyte PDGFR $\beta$, chemotaxis and TNFa production. Clin Immunol 2010;137:147-56.

57. Frostegård J, Svenungsson E, Wu R, Gunnarsson I, Lundberg IE, Klareskog $L$, et al. Lipid peroxidation is enhanced in patients with systemic lupus erythematosus and is associated with arterial and renal disease manifestations. Arthritis Rheum 2005;52:192-200.

58. Hansson GK, Libby P. The immune response in atherosclerosis: a double-edged sword. Nat Rev Immunol 2006;6:508-19.

59. Tripi LM, Manzi S, Chen Q, Kenney M, Shaw P, Kao A, et al. Relationship of serum paraoxonase 1 activity and paraoxonase 1 genotype to risk of systemic lupus erythematosus. Arthritis Rheum 2006;54:1928-39.

60. Marsillach J, Becker JO, Vaisar T, Hahn BH, Brunzell JD, Furlong CE, et al. Paraoxonase-3 is depleted from the high-density lipoproteins of autoimmune disease patients with subclinical atherosclerosis. J Proteome Res 2015;14:2046-54.

61. Dinu AR, Merrill JT, Shen C, Antonov IV, Myones BL, Lahita RG. Frequency of antibodies to the cholesterol transport protein apolipoprotein A1 in patients with SLE. Lupus 1998;7:355-60.

62. O'Neill SG, Giles I, Lambrianides A, Manson J, D'Cruz D, Schrieber $\mathrm{L}$, et al. Antibodies to apolipoprotein A-I, high-density lipoprotein, and C-reactive protein are associated with disease activity in patients with systemic lupus erythematosus. Arthritis Rheum 2010;62:84554.

63. Shoenfeld Y, Szyper-Kravitz M, Witte T, Doria A, Tsutsumi A, Tatsuya A, et al. Autoantibodies against protective molecules-C1q, C-reactive protein, serum amyloid $P$, mannose-binding lectin, and apolipoprotein A1: prevalence in systemic lupus erythematosus. Ann N Y Acad Sci 2007;1108:227-39.

64. Vuilleumier N, Reber G, James R, Burger D, de Moerloose P, Dayer $\mathrm{JM}$, et al. Presence of autoantibodies to apolipoprotein A-1 in patients with acute coronary syndrome further links autoimmunity to cardiovascular disease. J Autoimmun 2004;23:353-60.

65. Townsend D, Hughes E, Hussain R, Siligardi G, Baldock S, Madine $J$, et al. Heparin and methionine oxidation promote the formation of apolipoprotein A-I amyloid comprising a-helical and $\beta$-sheet structures. Biochemistry 2017;56:1632-44.

66. Chistiakov DA, Orekhov AN, Bobryshev YV. ApoA1 and ApoA1specific self-antibodies in cardiovascular disease. Lab Invest 2016;96:708-18.

67. Delgado Alves J, Kumar S, Isenberg DA. Cross-reactivity between anti-cardiolipin, anti-high-density lipoprotein and anti-apolipoprotein A-I IgG antibodies in patients with systemic lupus erythematosus and primary antiphospholipid syndrome. Rheumatology (Oxford) 2003;42:893-9.

68. Batuca JR, Ames PR, Isenberg DA, Alves JD. Antibodies toward high-density lipoprotein components inhibit paraoxonase activity in patients with systemic lupus erythematosus. Ann N Y Acad Sci 2007;1108:137-46.

69. Yu HH, Chen PC, Yang YH, Wang LC, Lee JH, Lin YT, et al. Statin reduces mortality and morbidity in systemic lupus erythematosus patients with hyperlipidemia: a nationwide population-based cohort study. Atherosclerosis 2015;243:11-8. 
70. Castejon R, Castañeda A, Sollet A, Mellor-Pita S, Tutor-Ureta P, Jimenez-Ortiz C, et al. Short-term atorvastatin therapy improves arterial stiffness of middle-aged systemic lupus erythematosus patients with pathological pulse wave velocity. Lupus 2017;26:355-64.

71. Orekhov AN, Misharin AY, Tertov W, Khashimov K, Pokrovsky SN, Repin VS, et al. Artificial HDL as an anti-atherosclerotic drug. Lancet 1984;2:1149-50.

72. Krause BR, Remaley AT. Reconstituted HDL for the acute treatment of acute coronary syndrome. Curr Opin Lipidol 2013;24:480-6.

73. Tardif JC, Grégoire J, L'Allier PL, Ibrahim R, Lespérance J, Heinonen $\mathrm{TM}$, et al. Effects of reconstituted high-density lipoprotein infusions on coronary atherosclerosis: a randomized controlled trial. JAMA 2007;297:1675-82.

74. Tardif JC, Grégoire J, L'Allier PL, Ibrahim R, Lespérance J, Heinonen $\mathrm{TM}$, et al. Effects of reconstituted high-density lipoprotein infusions on coronary atherosclerosis: a randomized controlled trial. JAMA 2007;297:1675-82.

75. Tricoci P, D'Andrea DM, Gurbel PA, Yao Z, Cuchel M, Winston B, et al. Infusion of reconstituted high-density lipoprotein, CSL112, in patients with atherosclerosis: safety and pharmacokinetic results from a phase 2 a randomized clinical trial. J Am Heart Assoc 2015;4:e002171.

76. CSL Behring, sponsor. Study to investigate CSL112 in subjects with acute coronary syndrome (AEGIS-II). Clinicaltrails.gov identifier: NCT03473223; 2018.

77. Morin EE, Guo L, Schwendeman A, Li XA. HDL in sepsis: risk factor and therapeutic approach. Front Pharmacol 2015;6:244.
78. Pajkrt D, Doran JE, Koster F, Lerch PG, Arnet B, van der Poll T, et al. Antiinflammatory effects of reconstituted high-density lipoprotein during human endotoxemia. J Exp Med 1996;184:1601-8.

79. Zhang Z, Datta G, Zhang Y, Miller AP, Mochon P, Chen YF, et al. Apolipoprotein A-I mimetic peptide treatment inhibits inflammatory responses and improves survival in septic rats. Am J Physiol Heart Circ Physiol 2009;297:H866-73.

80. Moreira RS, Irigoyen M, Sanches TR, Volpini RA, Camara NO, Malheiros DM, et al. Apolipoprotein A-I mimetic peptide 4F attenuates kidney injury, heart injury, and endothelial dysfunction in sepsis. Am J Physiol Regul Integr Comp Physiol 2014;307:R514-24.

81. Charles-Schoeman C, Banquerigo ML, Hama S, Navab M, Park GS, Van Lenten BJ, et al. Treatment with an apolipoprotein A-1 mimetic peptide in combination with pravastatin inhibits collagen-induced arthritis. Clin Immunol 2008;127:234-44.

82. Woo JM, Lin Z, Navab M, Van Dyck C, Trejo-Lopez Y, Woo KM, et al. Treatment with apolipoprotein A-1 mimetic peptide reduces lupuslike manifestations in a murine lupus model of accelerated atherosclerosis. Arthritis Res Ther 2010;12:R93.

83. Black LL, Srivastava R, Schoeb TR, Moore RD, Barnes S, Kabarowski $\mathrm{JH}$. Cholesterol-independent suppression of lymphocyte activation, autoimmunity, and glomerulonephritis by apolipoprotein A-I in normocholesterolemic lupus-prone mice. J Immunol 2015;195:4685-98.

84. Schwendeman A, Sviridov DO, Yuan W, Guo Y, Morin EE, Yuan Y, et al. The effect of phospholipid composition of reconstituted HDL on its cholesterol efflux and anti-inflammatory properties. J Lipid Res 2015;56:1727-37. 